الحمل الطبيعي لانتقال الحرارة في حيز مسامي مربع مع تشخين زاوي ومجال مغناطيسي

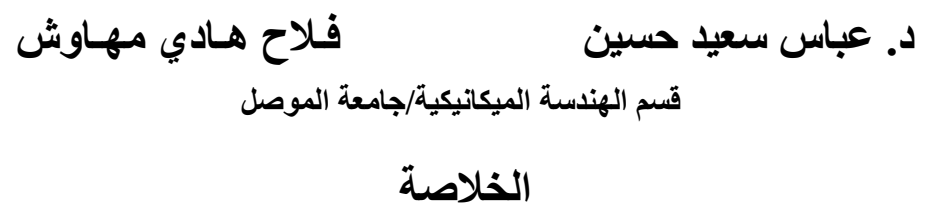

توثق هذه الدراسة العددية عملية انتقال الحرارة بالحمل الطبيعي في حيز مسامي مربع مع تسخين زاوي

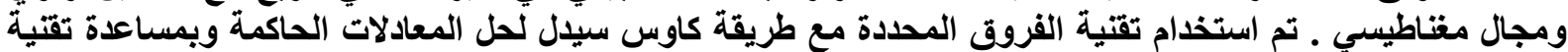
(Over Relaxation)

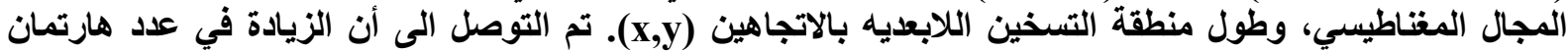

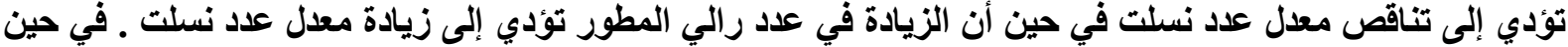

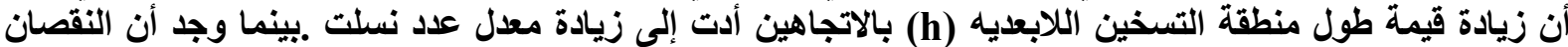

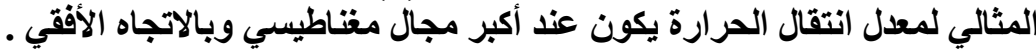

\title{
Natural Convection Heat Transfer in a Square Porous Enclosure with Corner Heating and Magnetic Field
}

\author{
Dr. Abbas Saeed Hussain Falah Hadi Mhawish \\ Department of Mechanical Engineering/ University of Mosul
}

\begin{abstract}
This numerical study documents the phenomena of heat transfer natural convection in a square porous cavity with corner heating and magnetic field. The finite difference technique with Gauss-Siedel method is used to solve the governing equations with aid of (Over Relaxation) technique of a range (1.1-1.3). The governing parameters are modified Rayleigh number, Hartmann's number, inclination angle of magnetic field and dimensionless length (non- dimensional heating region in both directions $(x, y))$. It was concluded that increase in the Hartman number leads to a decrease in the average Nusselt number while the increase in the modified Rayleigh number increases the average Nusselt number. The increase of dimensionless length (h) led to an increase the average Nusselt number. However, the optimum reducing of the heat transfer rate was obtained at a large magnetic field in the horizontal direction.
\end{abstract}

Keyword: Natural Convection, Square Porous, Corner Heating, Magnetic Field. 


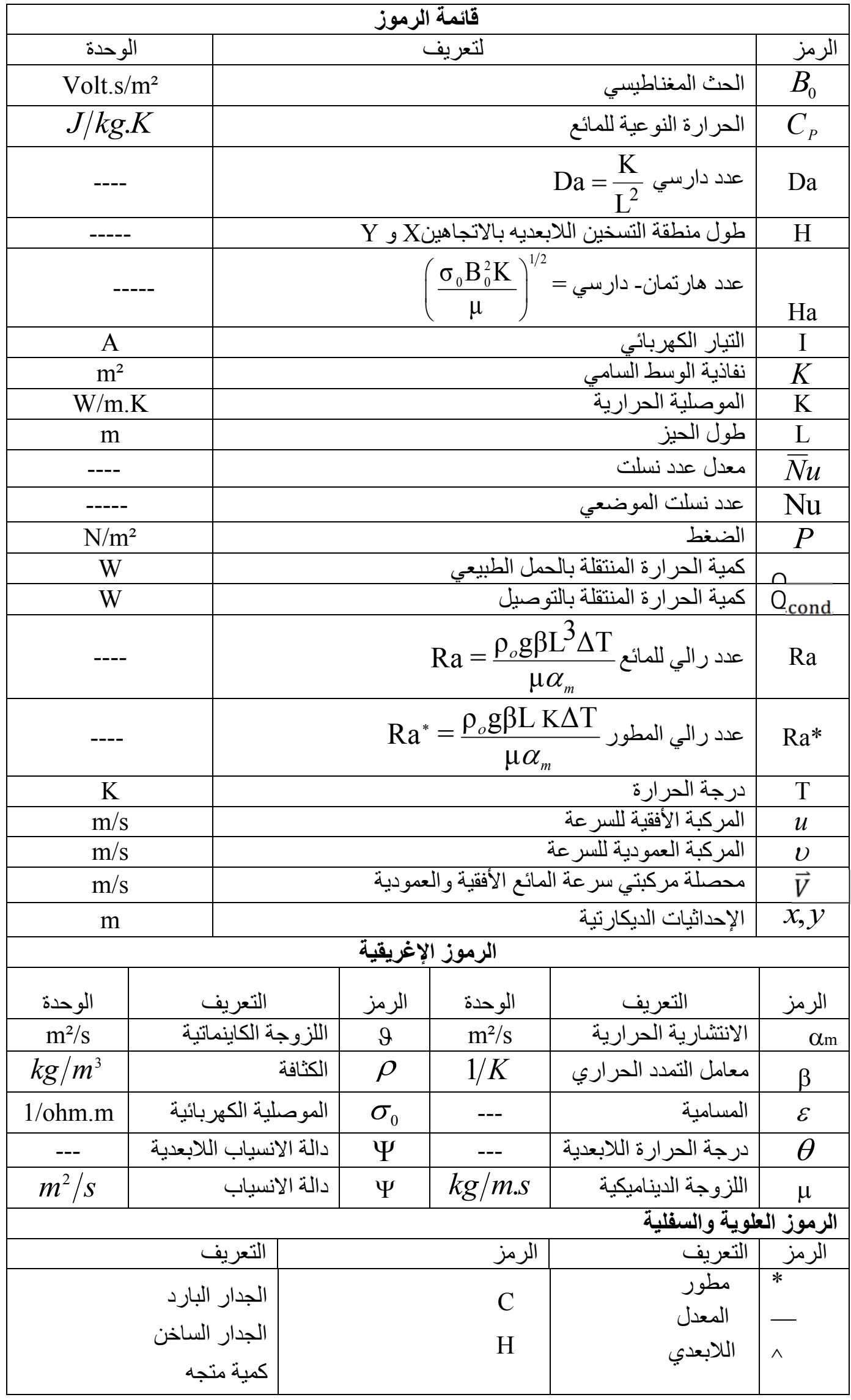




\section{1 المقدمة}

يحدث انتقال الحرارة بالحمل الطبيعي إذا ما وضع جسم في مائع عند درجة حرارة أعلى أو أوطأ من الجسم.

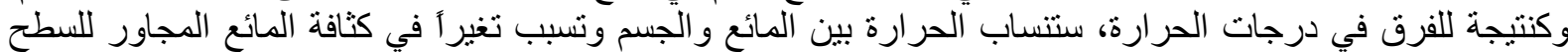

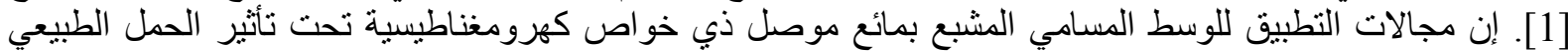

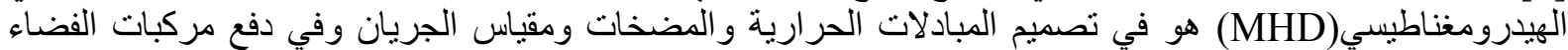

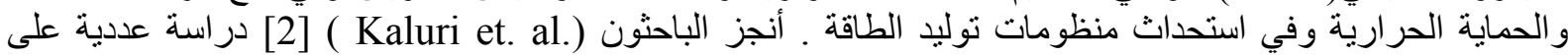

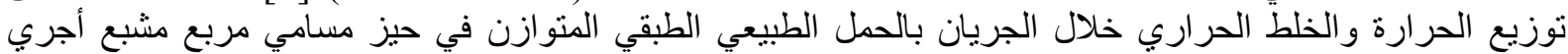

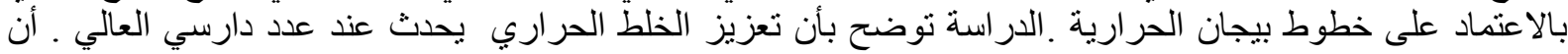

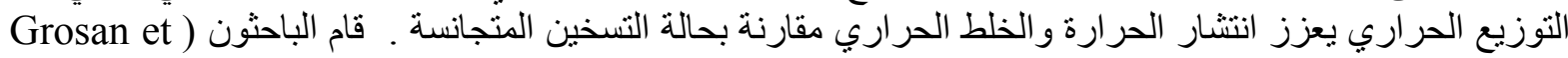

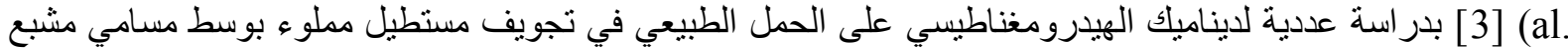

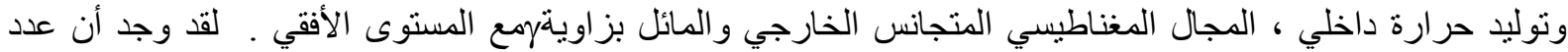

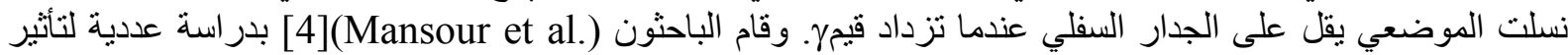

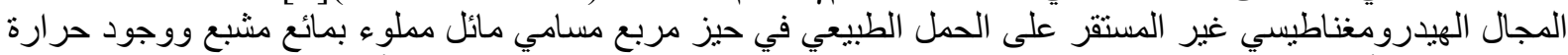

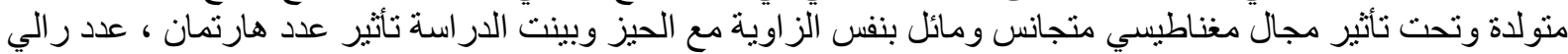

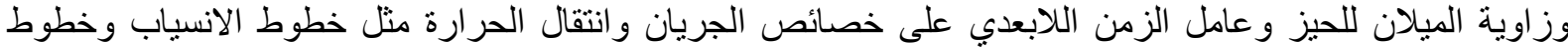

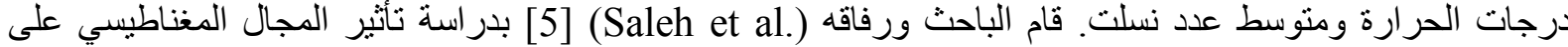

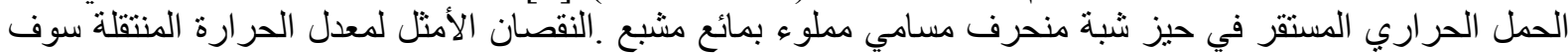

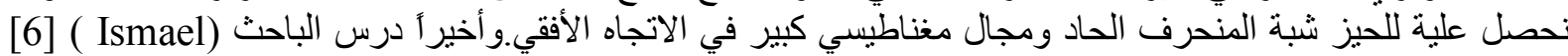

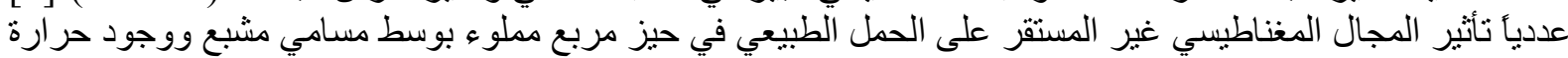

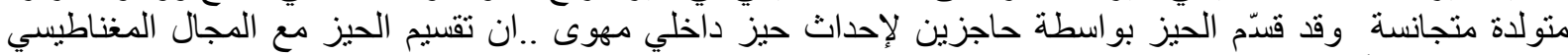
العمودي لهما تأثثر إخماد للجريان و انتقال الحيز بوال

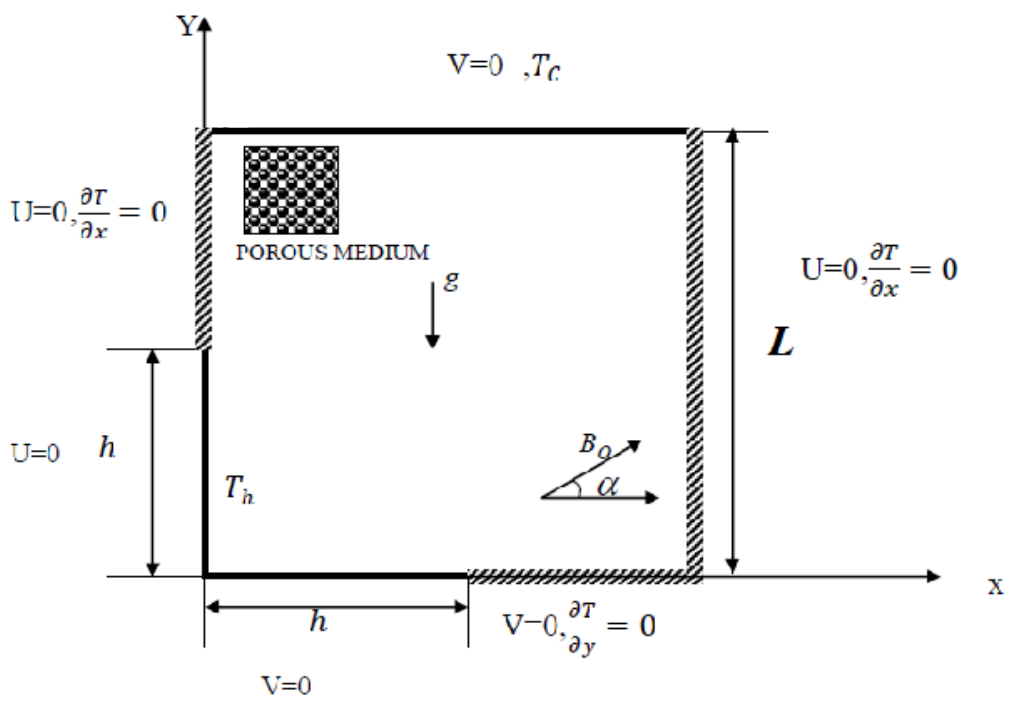

$$
\text { الشكل (1) : النموذج الهندسي لمسألة }
$$

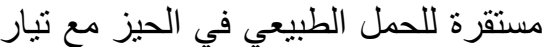

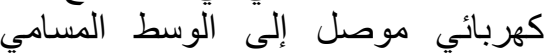

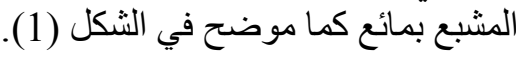

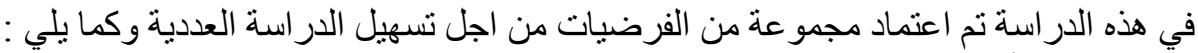

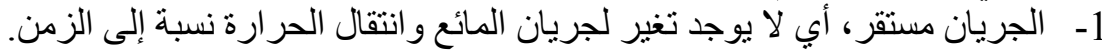

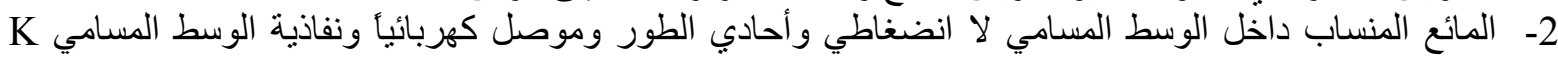
متساوية في جميع الاتجاهات.

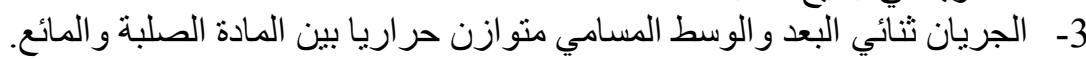
4- الوسط المسامي متجانس و المسامية ثابتة.

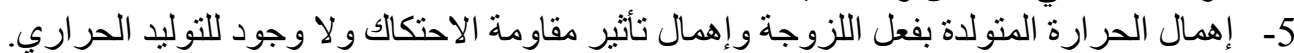

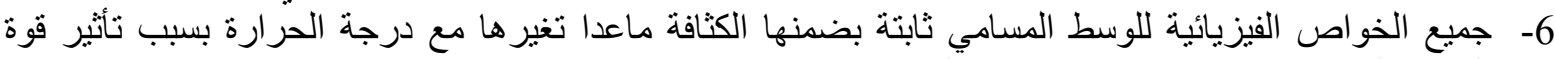
$\rho=\rho_{\circ}\left[1-\beta\left(T-T_{\circ}\right)\right]$ الطفو و التي يتم حسابها من تقريب بويسنسك الفئك 
7- - جدران الحيز المربع غير نفاذة .

8- القوة المغناطيسية تؤنثر باتجاه التعجيل الأرضي أو الأو الفقي أو مائلا .

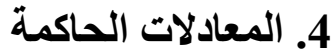

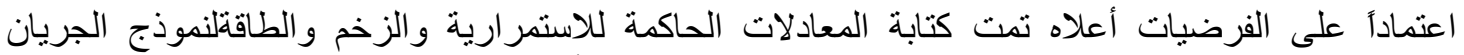

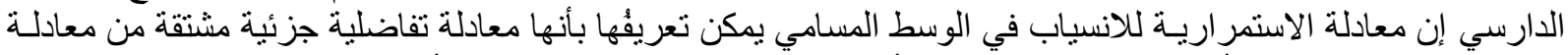

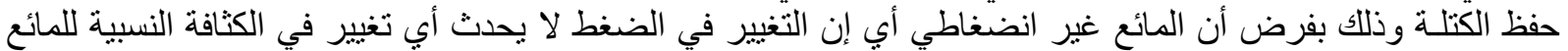

$\frac{\partial u}{\partial x}+\frac{\partial v}{\partial y}=0$

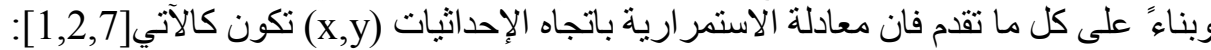

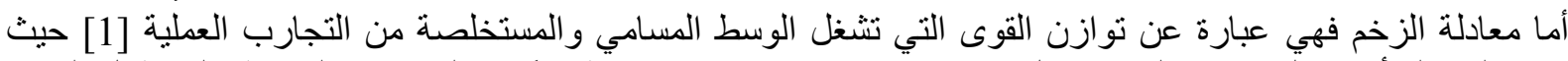

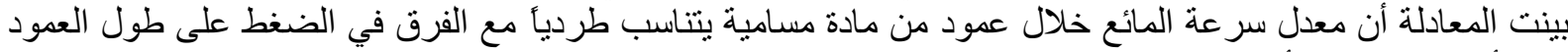

$\vec{V}=\frac{K}{\mu}\left(-\nabla \mathrm{P}+\rho \mathrm{g}+\mathrm{I} \times \mathrm{B}_{0}\right)$ مع الأخذ بالاعتبار التأثثر المغناطيسي المائل .

$\nabla . \mathrm{I}=0$

$\mathrm{I}=\sigma_{o}\left(-\nabla \varnothing+\mathrm{V} \times \mathrm{B}_{\mathrm{O}}\right)$

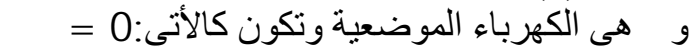

حيث تكون دائمأ الكهربائية معزولة في المحيط للحيز المغلق.وبذلك تصبح المعادلة (3) بعد التبسيط للاتجاه

$\mathrm{u}=-\frac{\mathrm{K}}{\mu} * \frac{\partial P}{\partial x}-\frac{\sigma_{o} B_{o} K}{\mu}\left(\mathrm{v} \sin \alpha * \cos \alpha-\mathrm{u}^{2} \sin ^{2} \alpha\right)$

وكذللك بالنسبة للاتجاه

$v=-\frac{\mathrm{K}}{\mu} * \frac{\partial P}{\partial y}-\frac{\sigma_{o} B_{o} K}{\mu}\left(\mathrm{u} \sin \alpha * \cos \alpha-\mathrm{vcos}^{2} \alpha\right)+\frac{\mathrm{K} \rho \mathrm{g}}{\mu}\left(\mathrm{T}-\mathrm{T}_{\mathrm{C}}\right)$

ولجعل الحل أسهل ، يمكن حذف حد الضغط من معادلة (6) و (7) عن طريق تفاضلوما ، الأولى نسبة إلى y و الثانيـة نسبة إلى X وسوف نحصل على معادلة الزخم :

$\frac{\partial u}{\partial y}-\frac{\partial v}{\partial x}=-\frac{\sigma_{O} K B_{O}}{\mu} * \frac{\partial v}{\partial y} \sin \alpha \cos \alpha+\frac{\partial u}{\partial y} * \frac{\sigma_{O} K B_{O}}{\mu} \sin ^{2} \alpha+$

$\frac{\partial u}{\partial x} * \frac{\sigma_{O} K B_{O}}{\mu} \sin \alpha \cos \alpha-\frac{\partial v}{\partial x} * \frac{\sigma_{O} K B_{O}}{\mu} \cos ^{2} \alpha-\frac{K \beta g}{\vartheta} * \frac{\partial T}{\partial x}$

أن معادلة الطاقة للوسط المسامي المتجانس (حيث كميـة الحر ارة المنتقلة تتمثل بالتوصيل و الحمل معا و عدم وجود توليد حر ارة وبإهمال حد اللزوجة) ، سوف تكون كالآتي [6] :

$u \frac{\partial T}{\partial x}+v \frac{\partial T}{\partial y}=\alpha_{m}\left[\frac{\partial^{2} T}{\partial x^{2}}+\frac{\partial^{2} T}{\partial y^{2}}\right]$

$$
\hat{\mathrm{X}}=\frac{\mathrm{x}}{\mathrm{L}}, \hat{\mathrm{Y}}=\frac{\mathrm{y}}{\mathrm{L}}, \quad \frac{T-T_{c}}{T_{h}-T_{c}} \quad \frac{\cdots}{\mathrm{m}}
$$

حيث تعرف المتغير ات اللابعدية كما يلي :

$\mathrm{Ha}=\mathrm{B}_{0} \sqrt{\frac{\sigma_{0} \mathrm{~K}}{\mu}} \quad, \quad \mathrm{Ra}^{*}=\frac{\rho_{O} g \beta K \Delta \mathrm{T}}{\mu \alpha_{m}}$ 
A t $\hat{Y}=1,0<\hat{X}<1, U=V=0, \Psi=0, \theta=0$

At $\hat{Y}=0,0 \leq \hat{X} \leq \mathrm{h}, \mathrm{U}=\mathrm{V}=0, \Psi=0, \theta=1$

A t $\hat{Y}=0, h<\hat{X} \leq 1, U=V=0, \frac{\partial \theta}{\partial \hat{Y}}=0$

A t $\hat{X}=0,0 \leq \hat{Y} \leq h, U=V=0, \Psi=0, \theta=1$

At $\hat{X}=0, h<\hat{X} \leq 1, U=V=0, \Psi=0, \frac{\partial \theta}{\partial \hat{X}}=0$

A t $\hat{X}=1,0 \leq \hat{Y} \leq 1, U=V=0, \Psi=0, \frac{\partial \theta}{\partial \hat{X}}=0$

$\overline{N u}=\int_{0}^{1}\left(\frac{\partial \theta}{\partial \hat{Y}}\right) \cdot d x$

ويعرف معدل عدد نسلت :

استخدمت تقنية الفرق المحدد لحل المعادلات الحاكمة (8) و (9) مع الظروف الحدية ـ بالاستعانة بطريقة كاوس

5.التحليل العددي سيدل التكر ارية وبدقة:

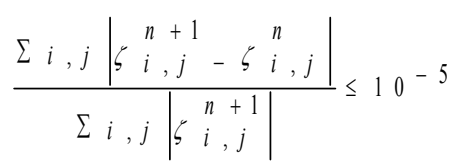

حيث تمثل ك مره T أو W وتمثل (n) عدد المحاو لات للبرنامج .للتأكد من صحة الحل سوف تتم مقارنة النتائج التي سيتم

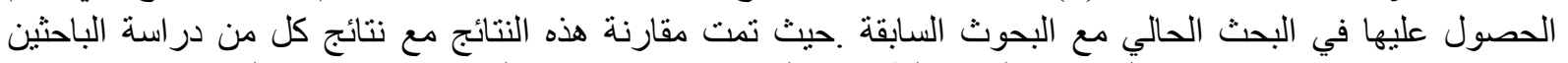
[8smaeel)

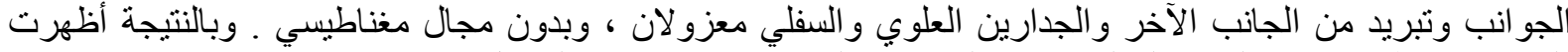

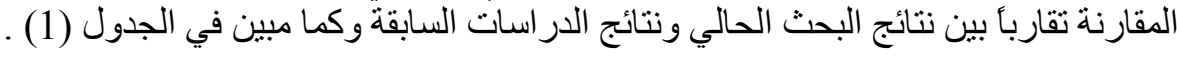

\begin{tabular}{|c|c|c|c|c|c|c|c|}
\multicolumn{2}{c|}{$(\mathrm{Ha}=0)(\mathrm{Ra})=50,100)$} \\
\hline \multirow{2}{*}{$R a^{*}$} & $\begin{array}{c}\text { Chan } \\
{[10]}\end{array}$ & $\begin{array}{c}\text { Burns } \\
{[11]}\end{array}$ & $\begin{array}{c}\text { Bejan\&Tien } \\
{[9]}\end{array}$ & $\begin{array}{c}\text { Bejan } \\
{[12]}\end{array}$ & $\begin{array}{c}\text { Dawood } \\
{[13]}\end{array}$ & $\begin{array}{c}\text { Ismaeel } \\
{[8]}\end{array}$ & $\begin{array}{c}\text { Present } \\
\text { Work }\end{array}$ \\
\hline 50 & 2.1 & 2.2 & 2.12 & 1.897 & 2.22 & 2.034 & 2.042 \\
\hline 100 & 3.54 & 3.6 & 3.25 & 3.433 & 3.472 & 3.472 & 3.218 \\
\hline
\end{tabular}

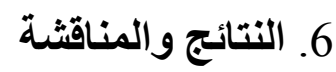

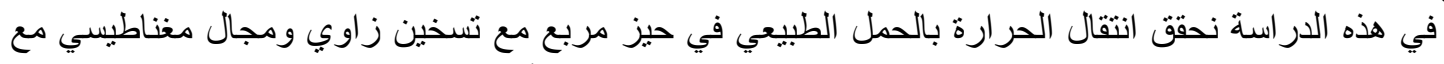

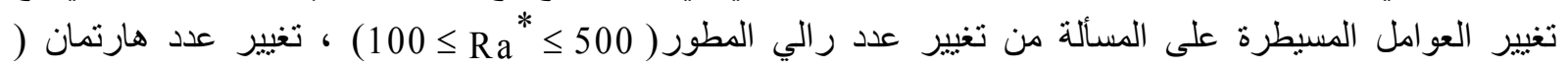

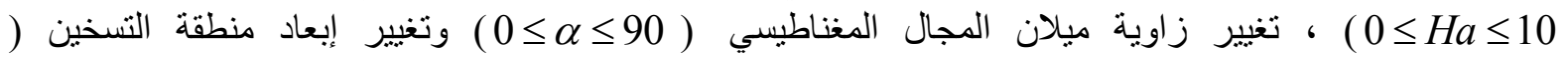

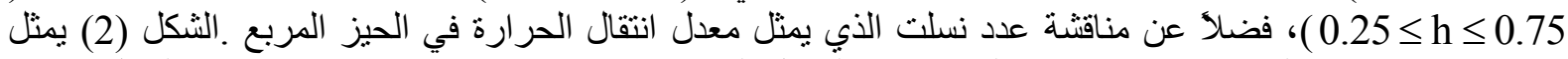

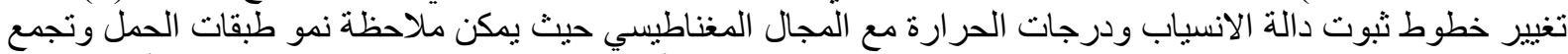

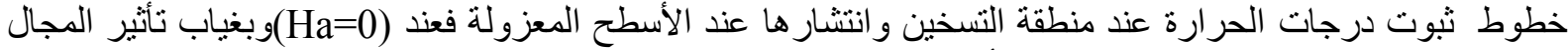

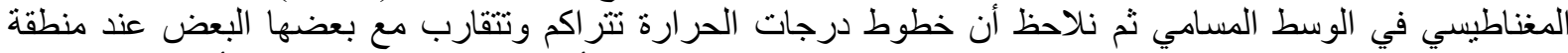

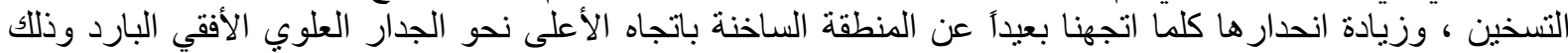

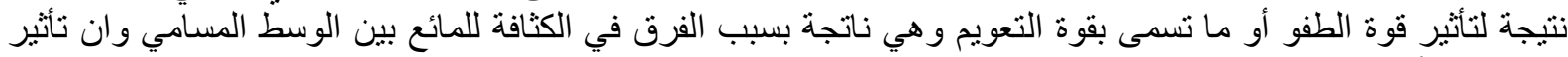

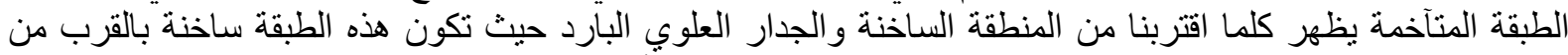

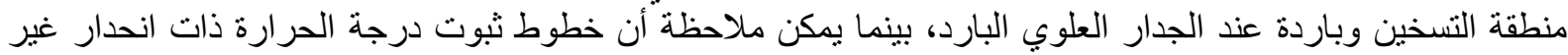

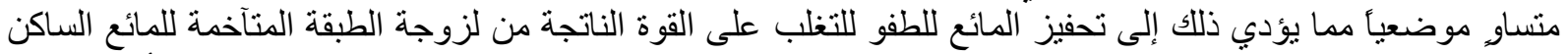

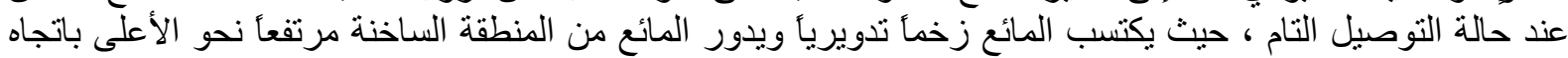
الجدار البارد ، 
No. 5

Dec. 2014

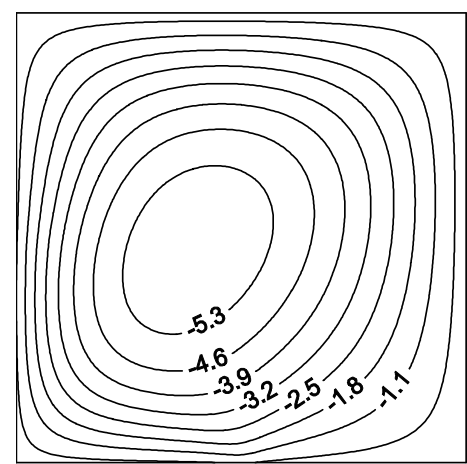

(a) $\mathrm{Ha}=0$
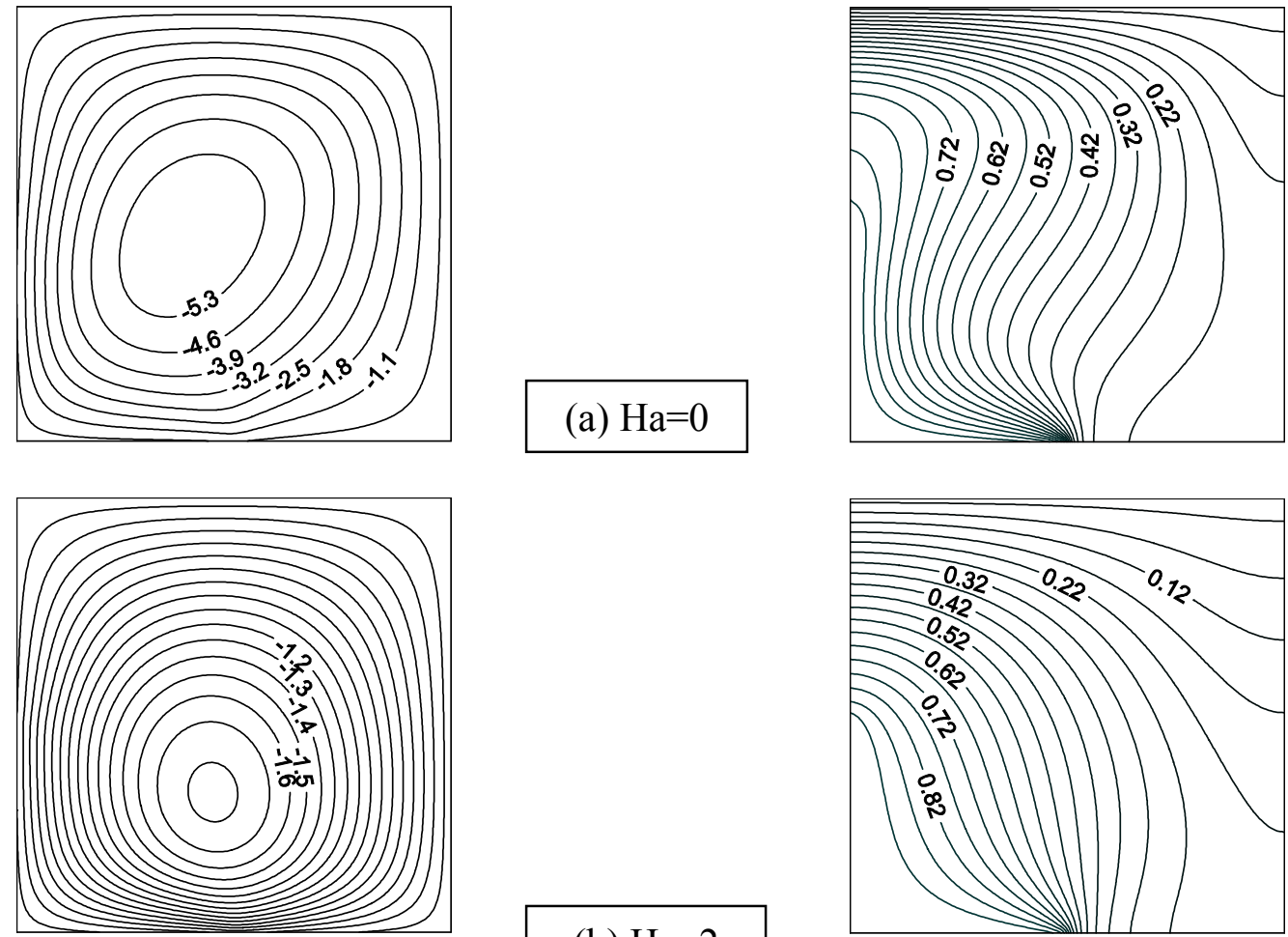

(b) $\mathrm{Ha}=2$
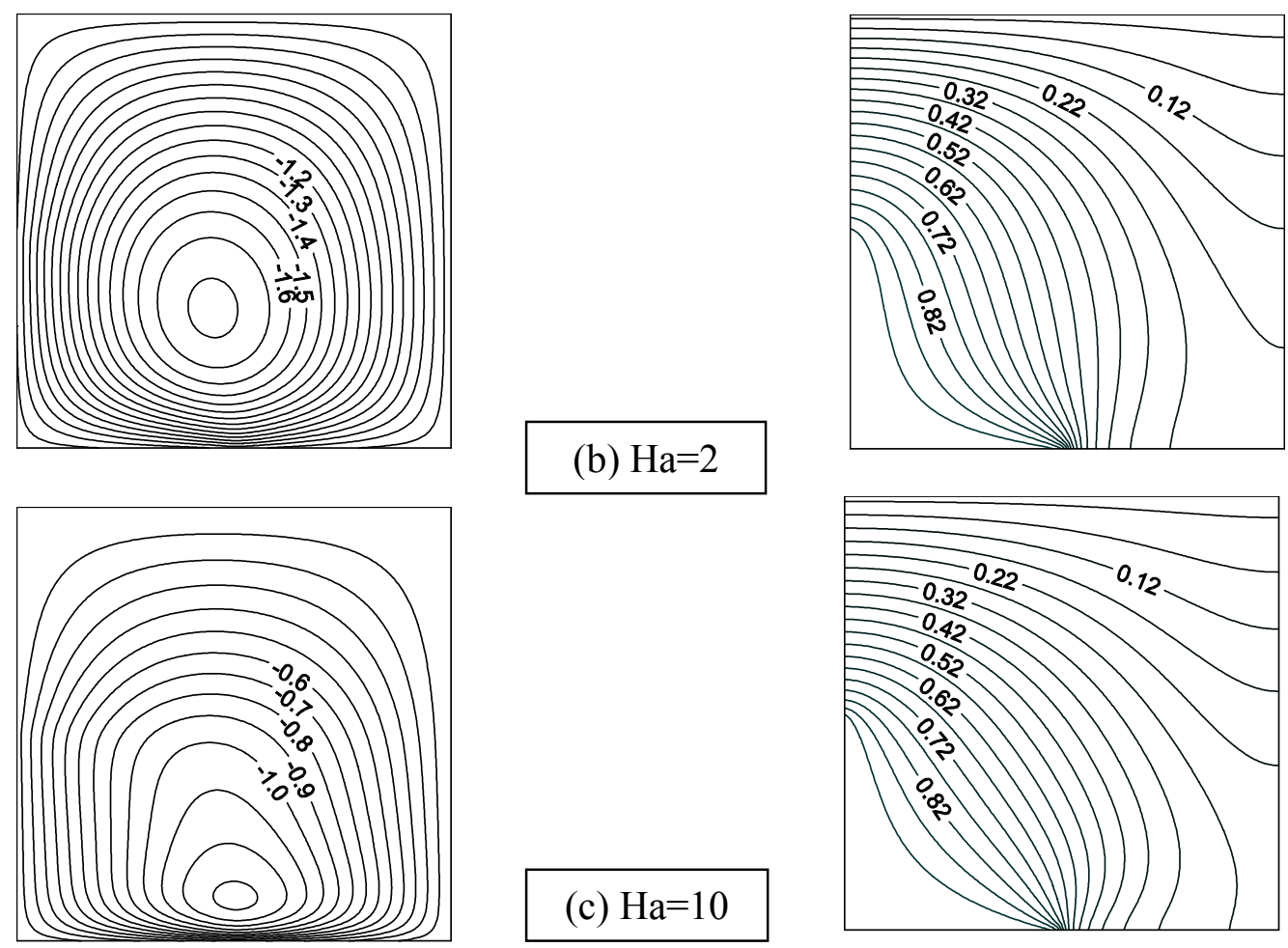

(c) $\mathrm{Ha}=10$

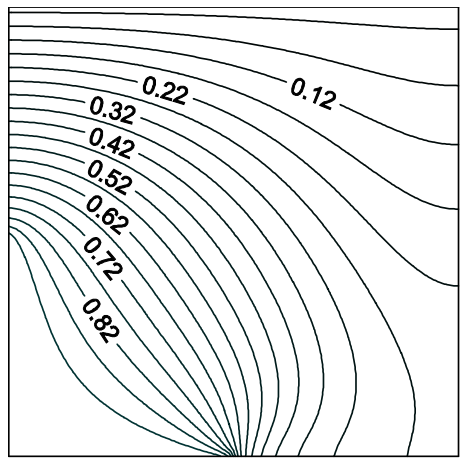

Ra*=100, الثكل (2) تغير خطوط ثبوت دالة الانسياب ودرجات الحرارة مع المجال المغناطيسي عند = (0,h (0.5)

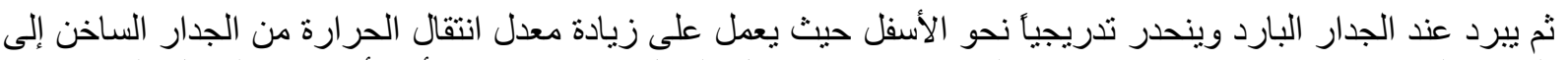

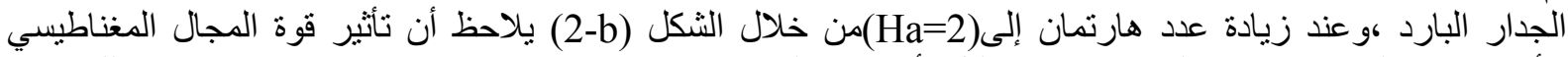

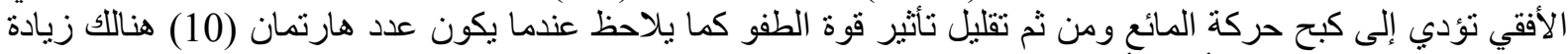

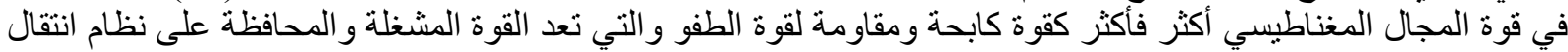

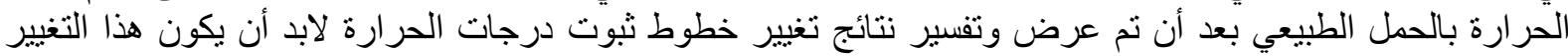
مصحوبأ بتغيير دالة الانسياب 
حسين: الحمل الطبيعي لانتقال الحرارة في حيز مسامي مربع مع تسخين زاوي ومجال مغناطيسي
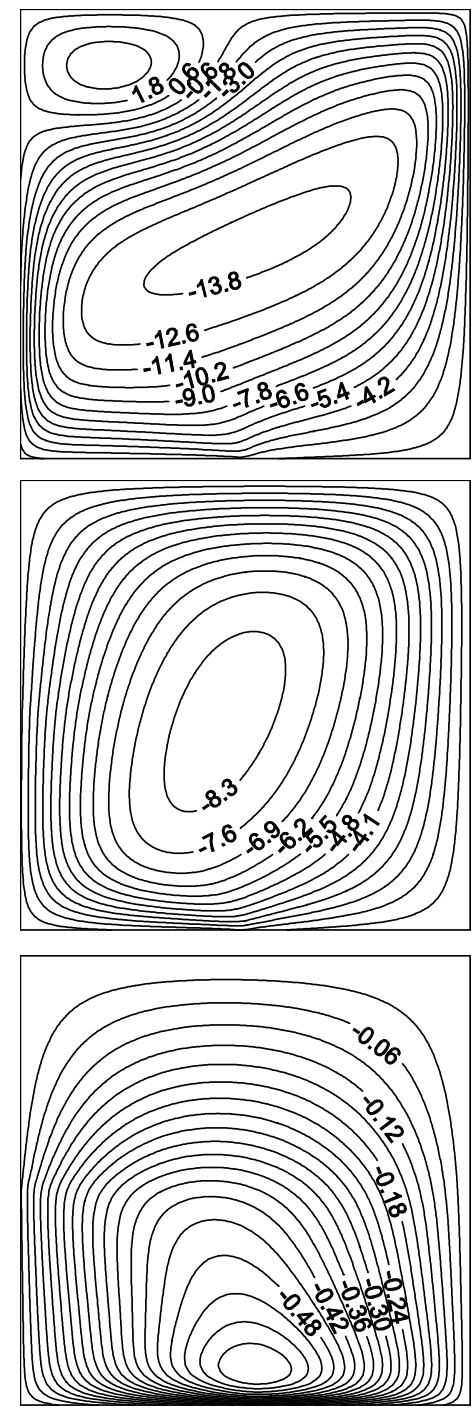

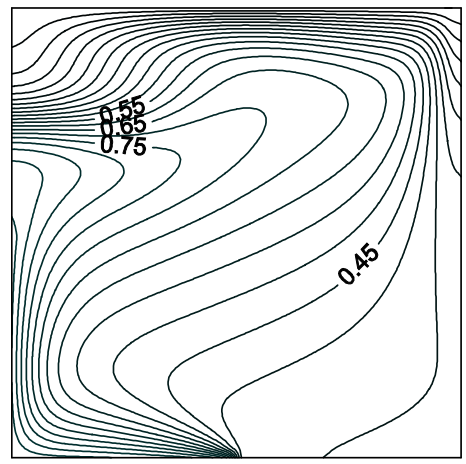

(b) $\mathrm{Ha}=2$
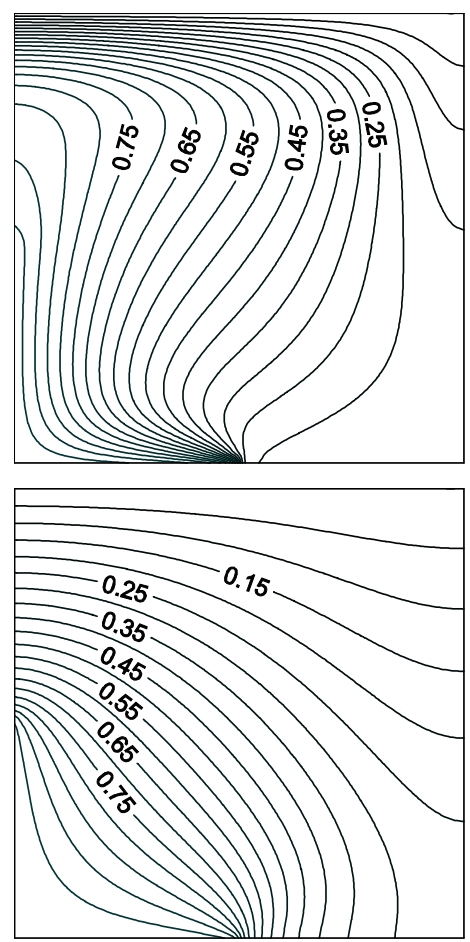

الثكل(3) تغير خطوط ثبوت دالة الانسياب ودرجات الحرارة مع المجال المغناطيسي عند

( $\mathrm{Ra}^{*}=500, \alpha=0, \mathrm{~h}=0.5$ )

في الثكل (a -2) عند عدد هارتمان (Ha=0) يمكن ملاحظة أن الجريان الطبقي الدراسي للمائع يبدأ بالنمو و التكوين

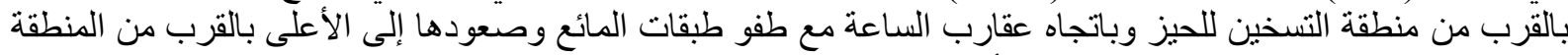

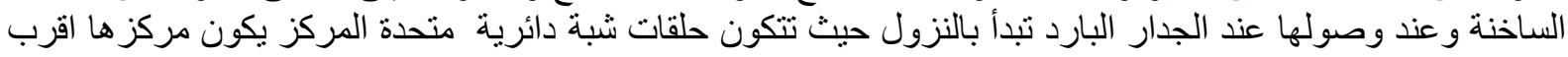

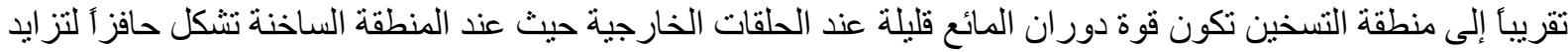

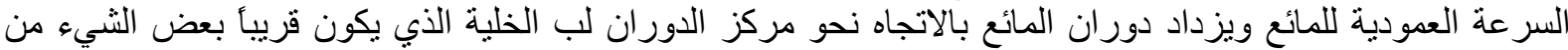

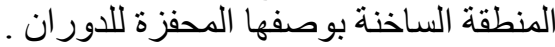



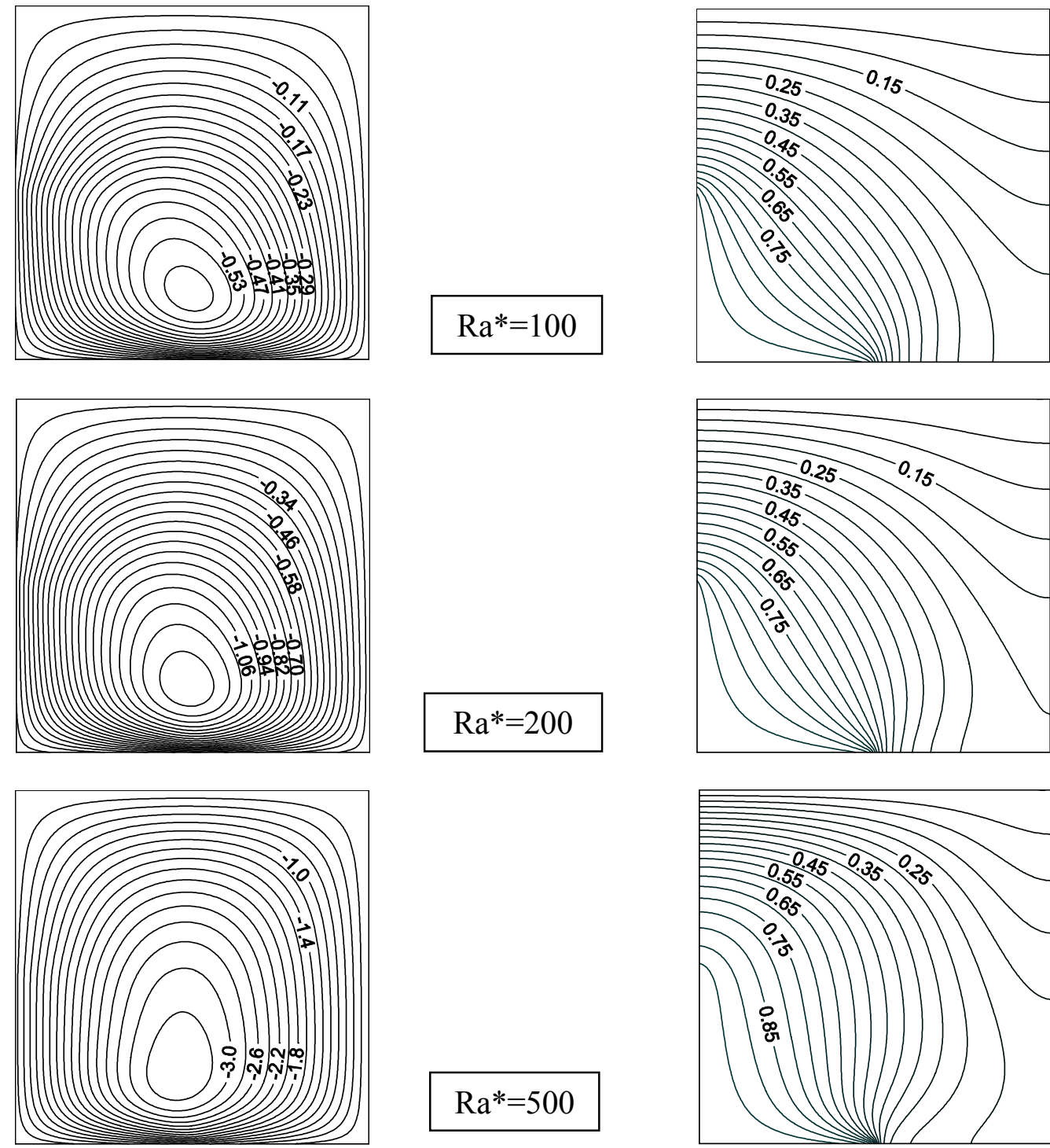

\section{$\mathrm{Ra}^{*}=500$}

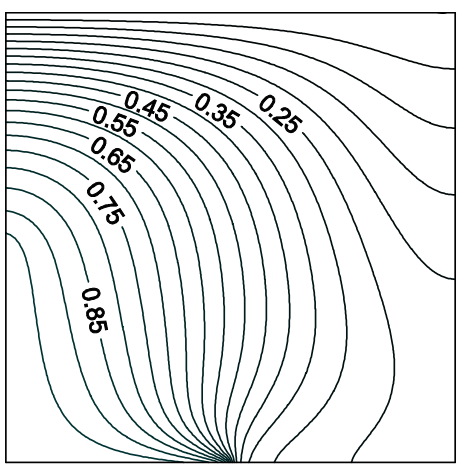

الثكل (4) تغير خطوط ثبوت دالة الانسياب ودرجات الحرارة مع عدد رالي المطور عند

$$
(\mathrm{Ha}=4, \alpha=0, \mathrm{~h}=0.5)
$$

عند زيادة عدد هارتمان (Ha=2)يلاحظ انكماش خطوط ثبوت دالة الانسياب وذللك بسبب زيادة قوة الكبح للمجال

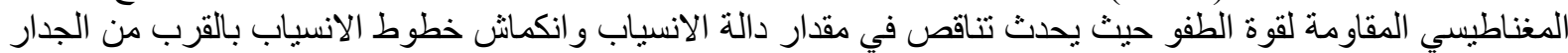

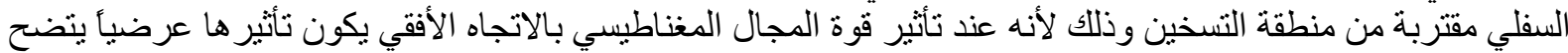

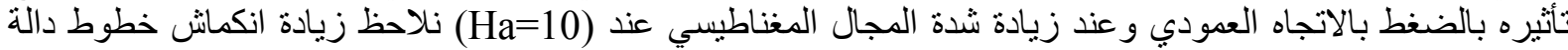

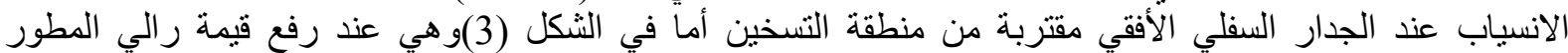

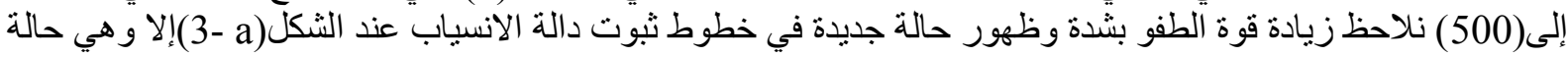

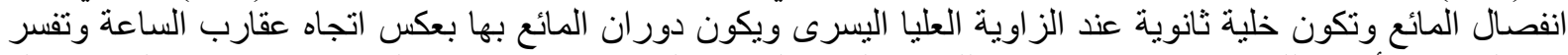

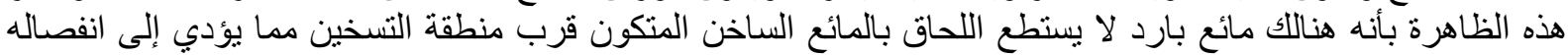

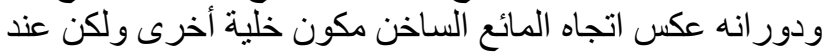


حسين: الحمل الطبيعي لانتقال الحرارة في حيز مسامي مربع مع تسخين زاوي ومجال مغناطيسي

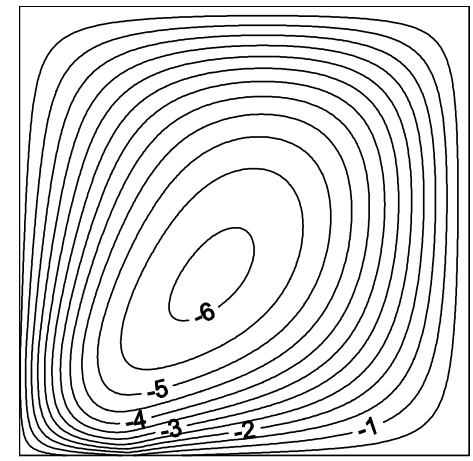

(a) $\mathrm{h}=0.25$
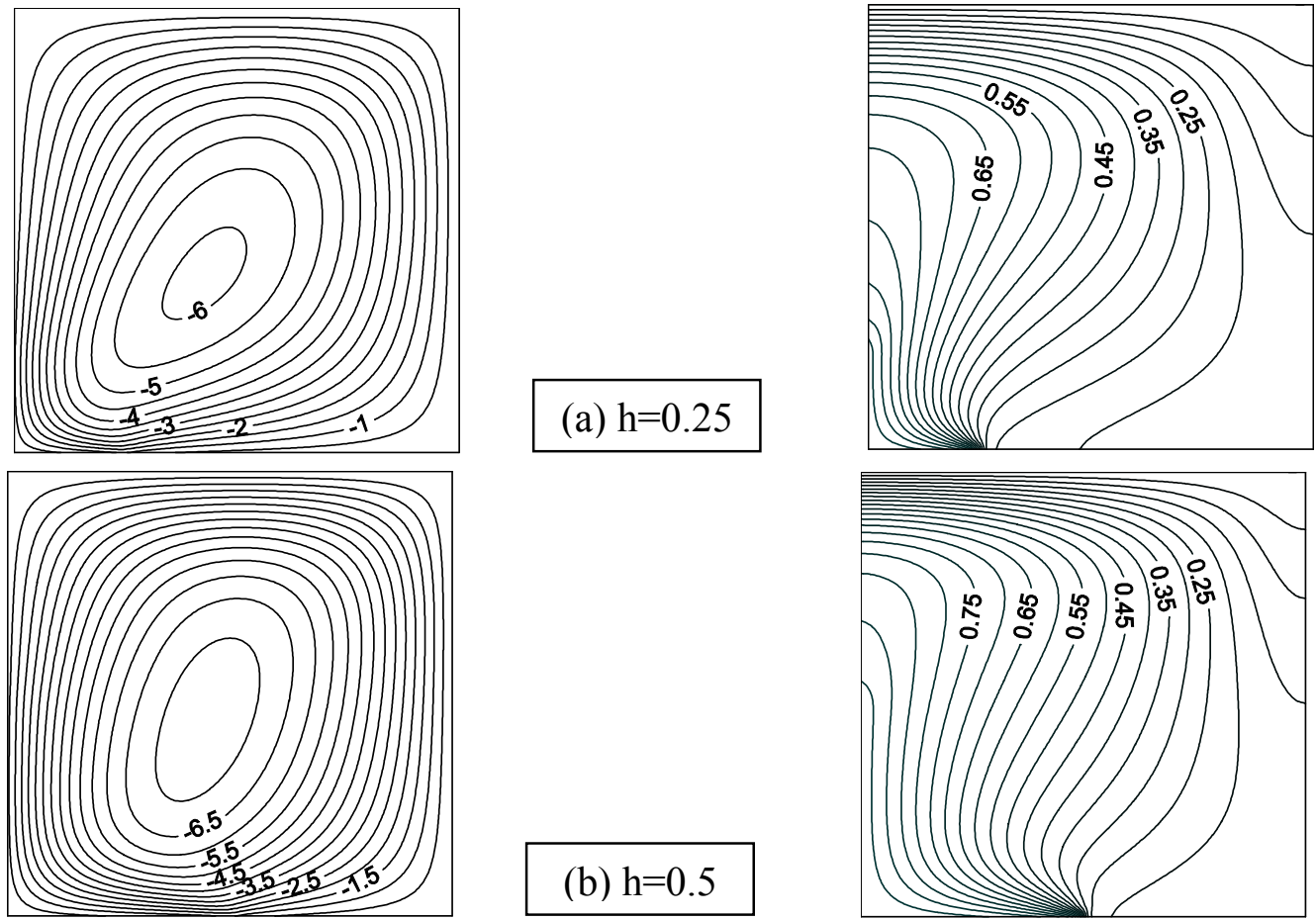

(b) $\mathrm{h}=0.5$
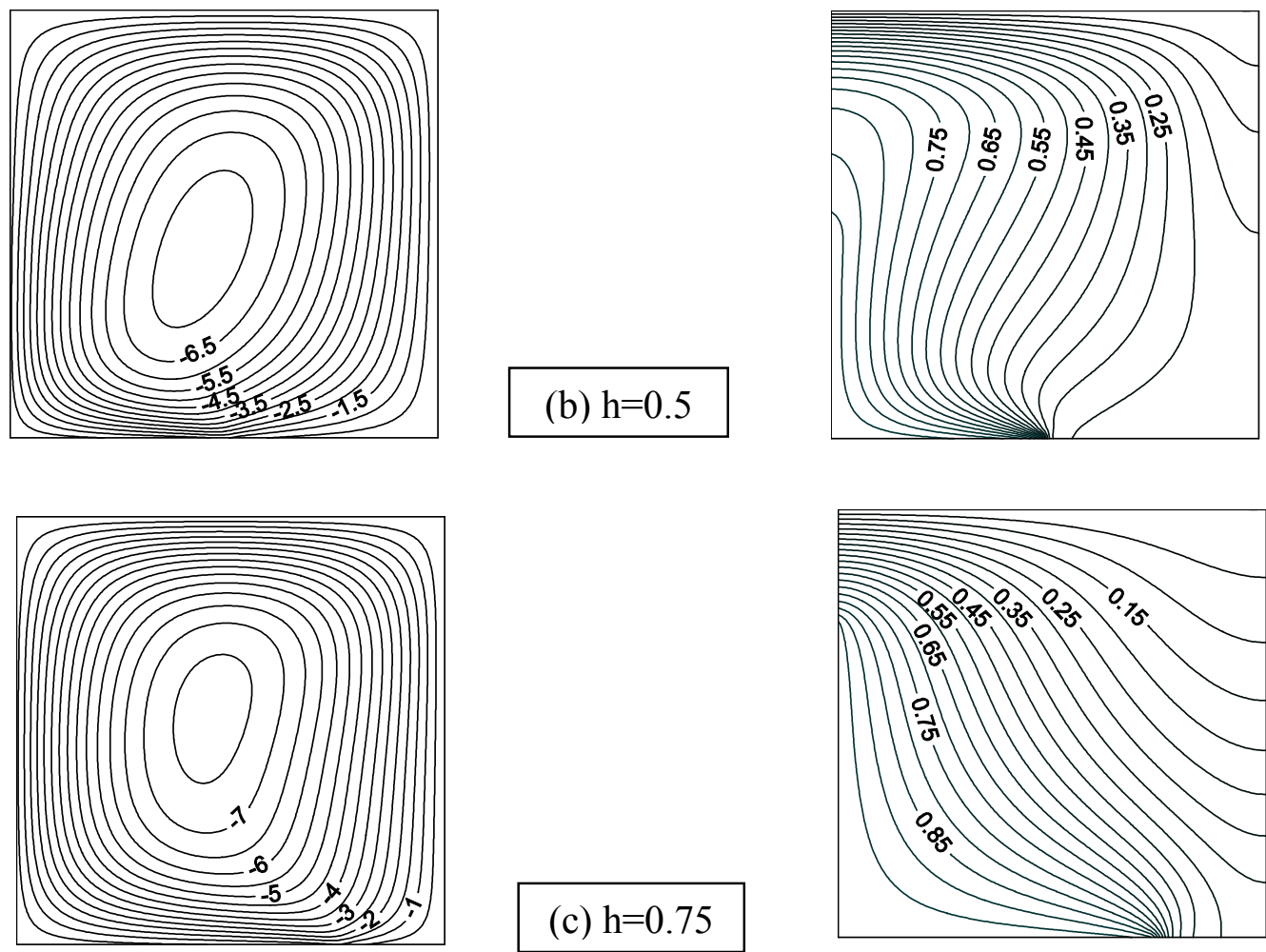

(c) $\mathrm{h}=0.75$

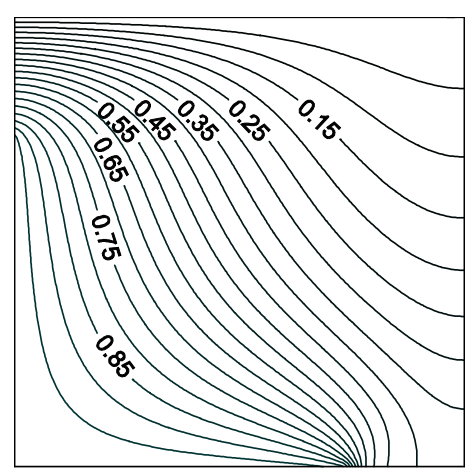

الثكل (5) تغير خطوط ثبوت دالة الانسياب ودرجات الحرارة مع أبعاد مختلفة لمنطقة التسخين عند) (c)

$$
\left(\mathrm{Ra}^{*}=400, \mathrm{Ha}=2, \alpha=0\right.
$$

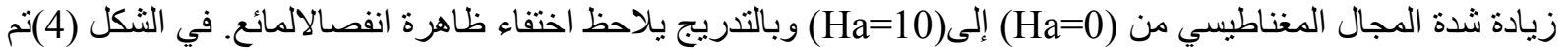

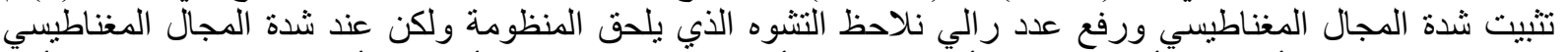

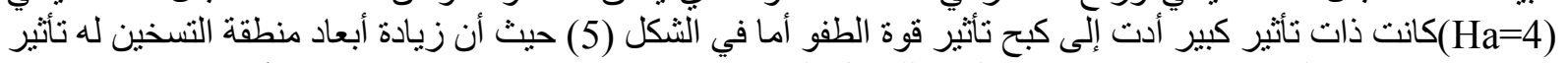

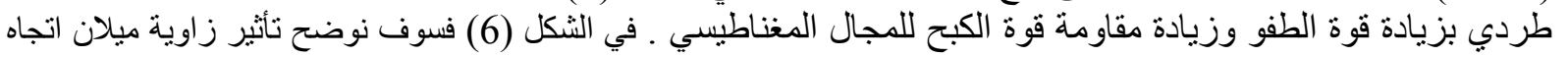

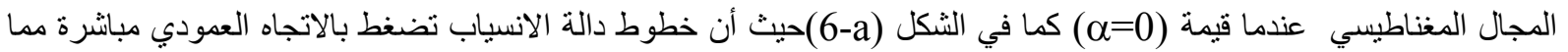

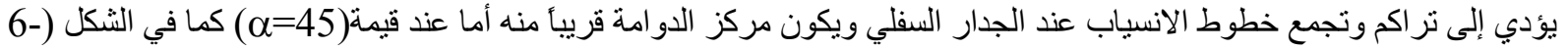

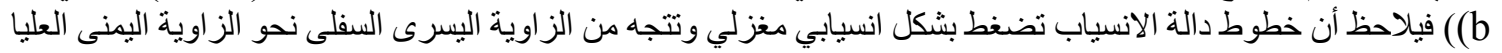



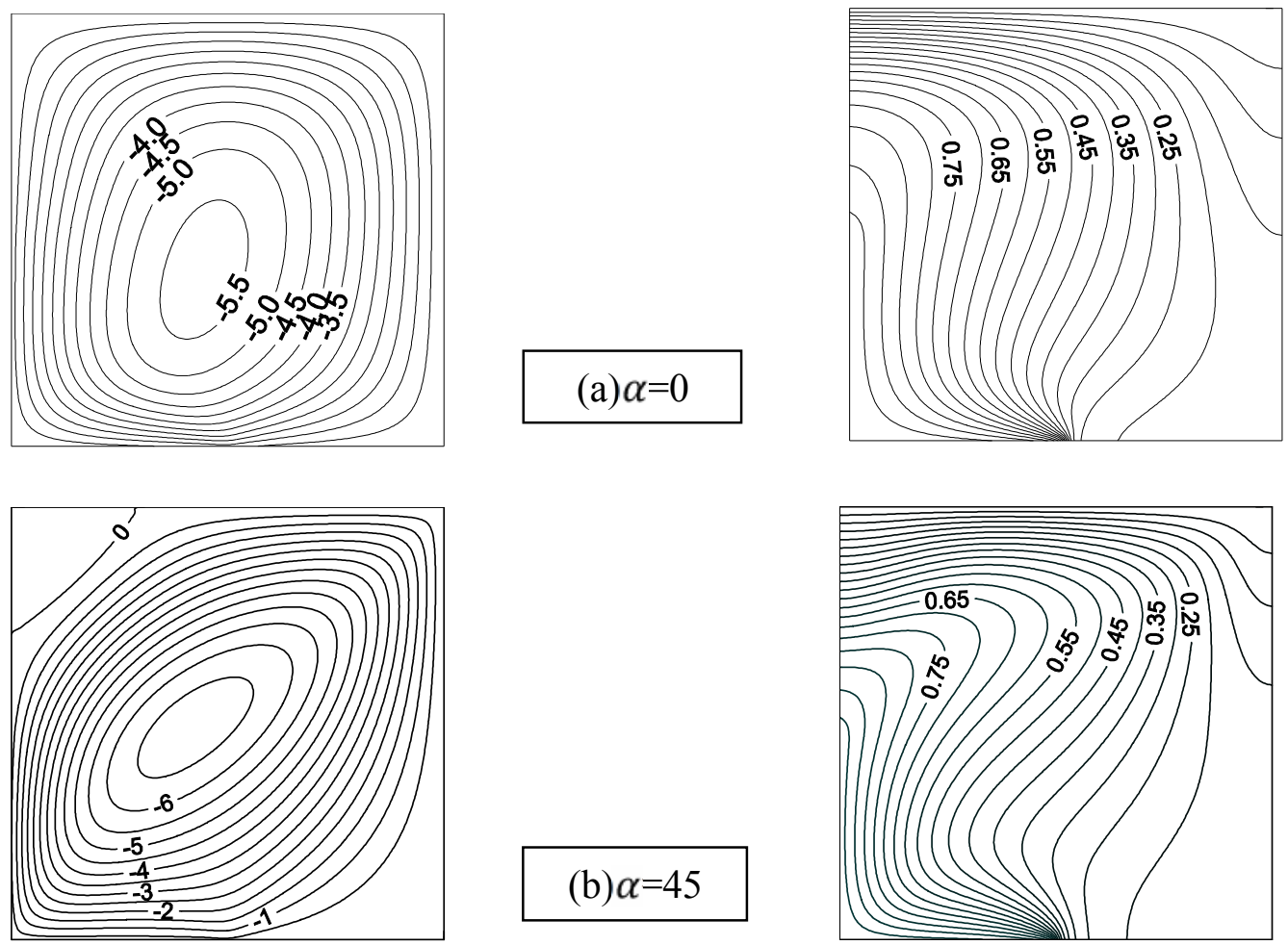

\section{(b) $\alpha=45$}
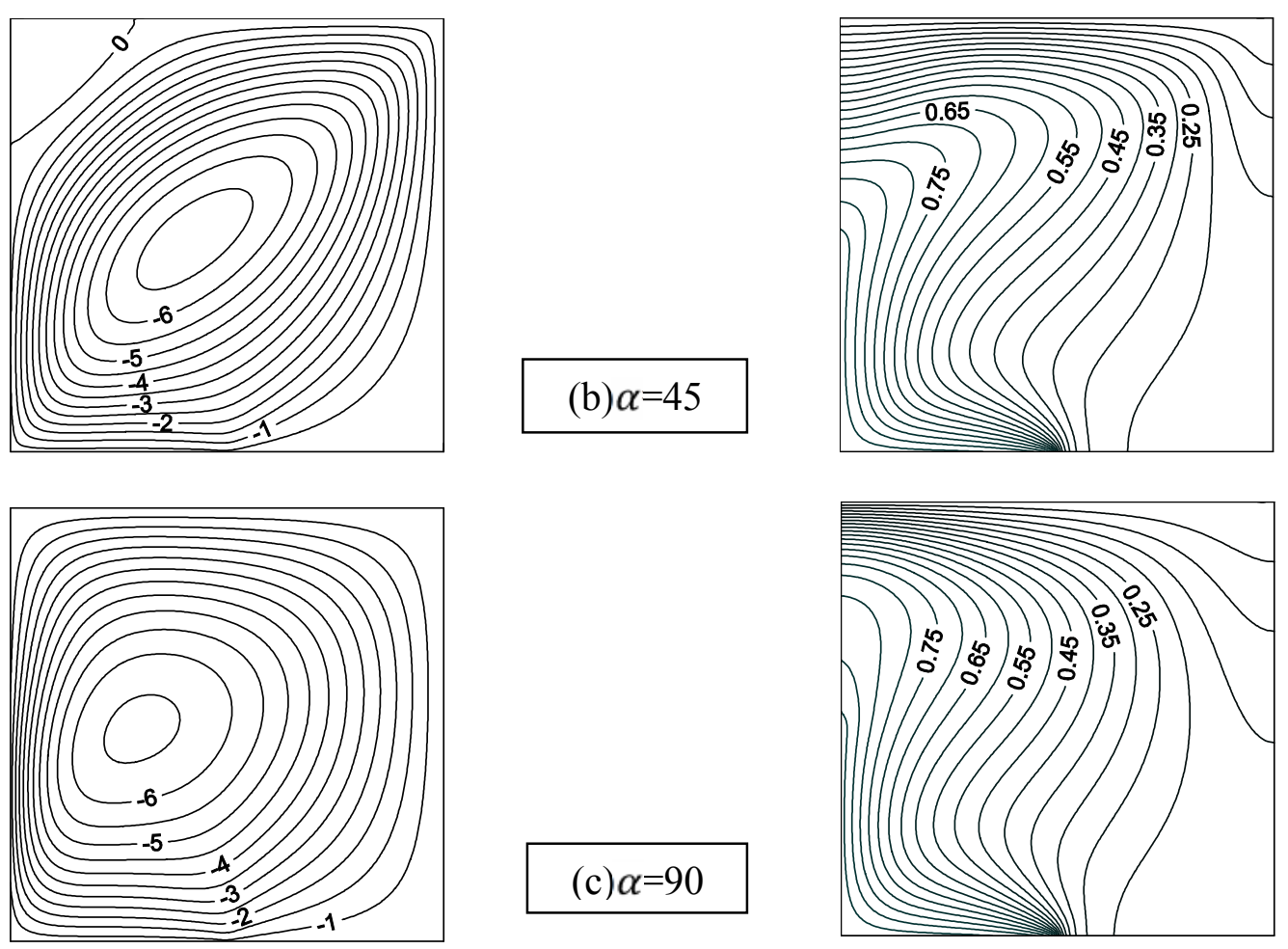

h=0.5Ha=2, الثكل (6) تغير خطوط ثبوت دالة الانسياب ودرجات الحرارة مع تغيير زاوية المجال المغناطيسي عند $\left(\mathrm{Ra}^{*}=300\right.$,

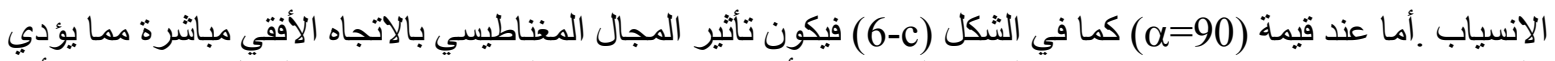

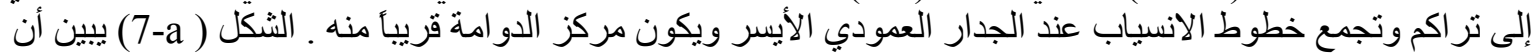

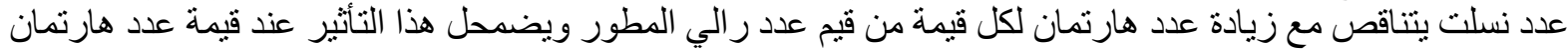

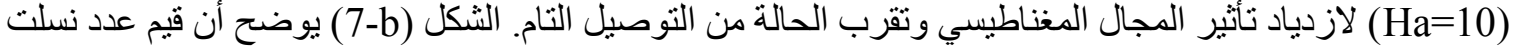

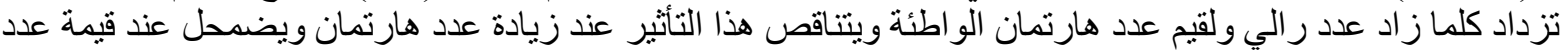

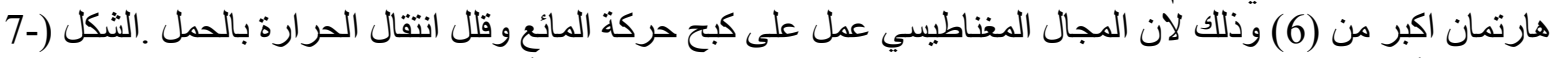

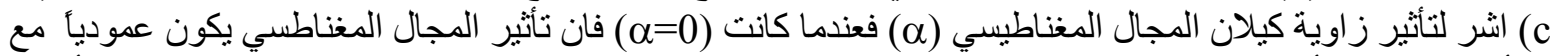

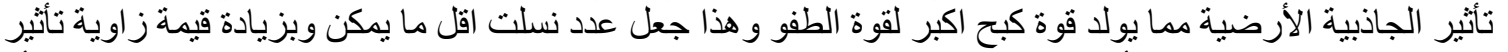

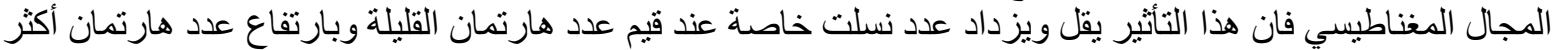

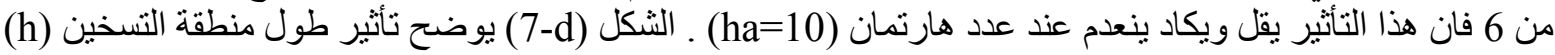


فبزيادتها يزداد عدد نسلت لان مساحة المنطقة المعرضة للتسخين كانت اكبر و أدت إلى زيادة كمية الحرارة المجهزة

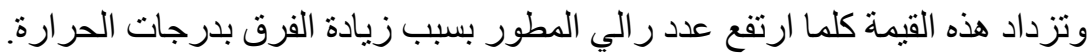
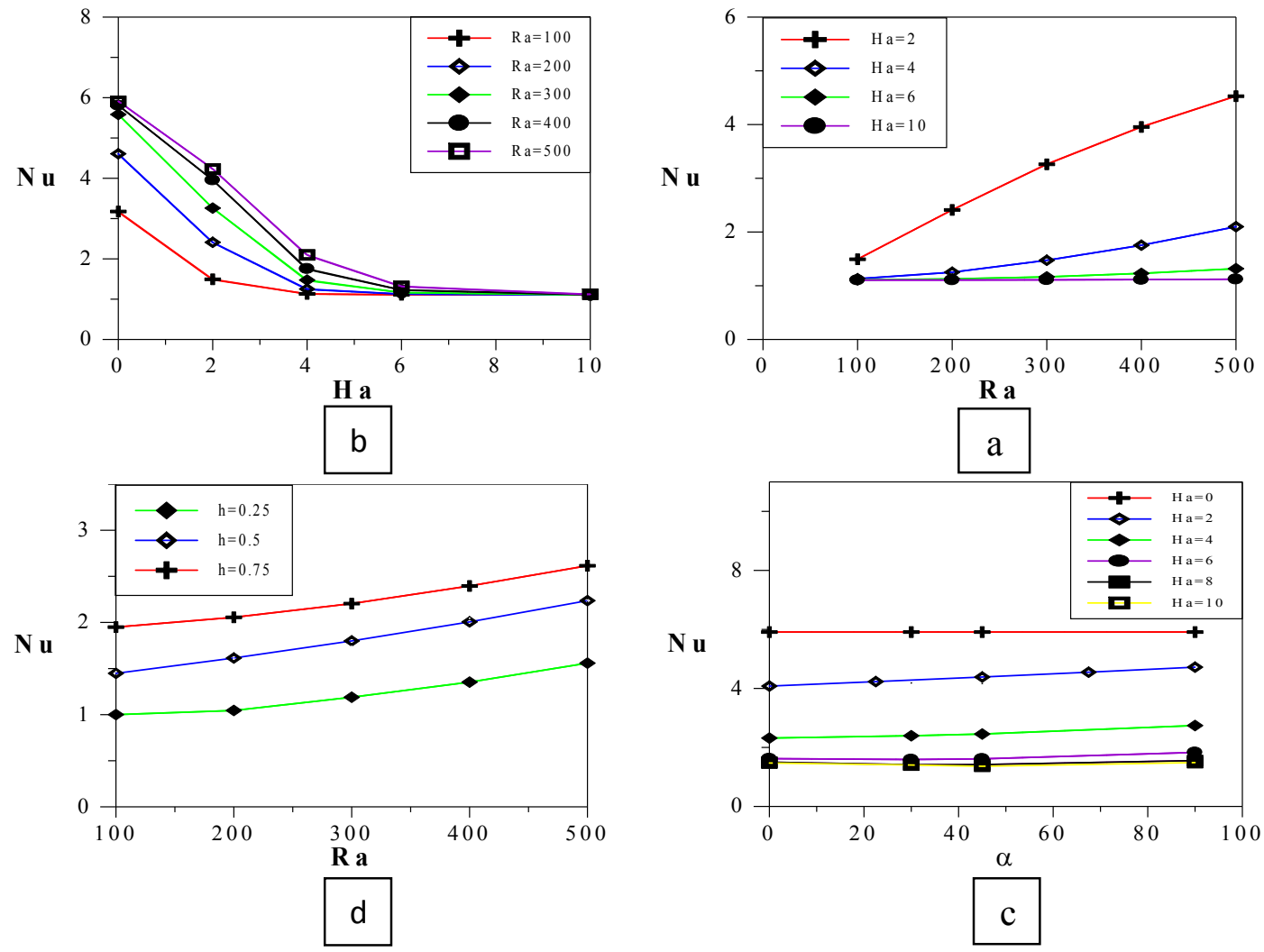

الثكل (7) تغير قيم عدد نسلت

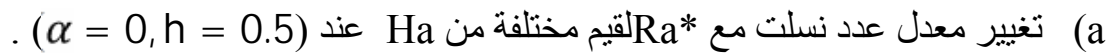

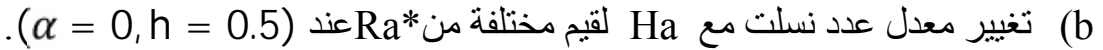

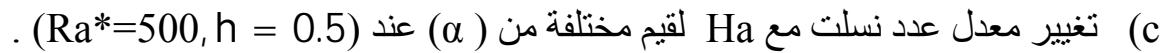

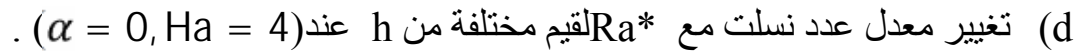

الجدول (2) النسب المئوية لانخفاض معدل عدد نسلت ما بين Ha=0=10 Ha

\begin{tabular}{|c|c|c|c|}
\hline$\alpha$ & $\mathrm{h}$ & $\mathrm{Ra}^{*}=100$ & $\mathrm{Ra}^{*}=500$ \\
\hline \multirow{3}{*}{0} & 0.25 & $315 \%$ & $600 \%$ \\
\cline { 2 - 4 } & 0.50 & $288 \%$ & $527 \%$ \\
\cline { 2 - 4 } & 0.75 & $225 \%$ & $479 \%$ \\
\hline \multirow{3}{*}{30} & 0.25 & $315 \%$ & $590 \%$ \\
\cline { 2 - 4 } & 0.50 & $287 \%$ & $522 \%$ \\
\cline { 2 - 4 } & 0.75 & $225 \%$ & $477 \%$ \\
\hline \multirow{3}{*}{45} & 0.25 & $315 \%$ & $584 \%$ \\
\cline { 2 - 4 } & 0.50 & $287 \%$ & $518 \%$ \\
\cline { 2 - 4 } & 0.75 & $225 \%$ & $477 \%$ \\
\hline \multirow{3}{*}{90} & 0.25 & $315 \%$ & $600 \%$ \\
\cline { 2 - 4 } & 0.50 & $287 \%$ & $515 \%$ \\
\cline { 2 - 4 } & 0.75 & $225 \%$ & $469 \%$ \\
\hline
\end{tabular}



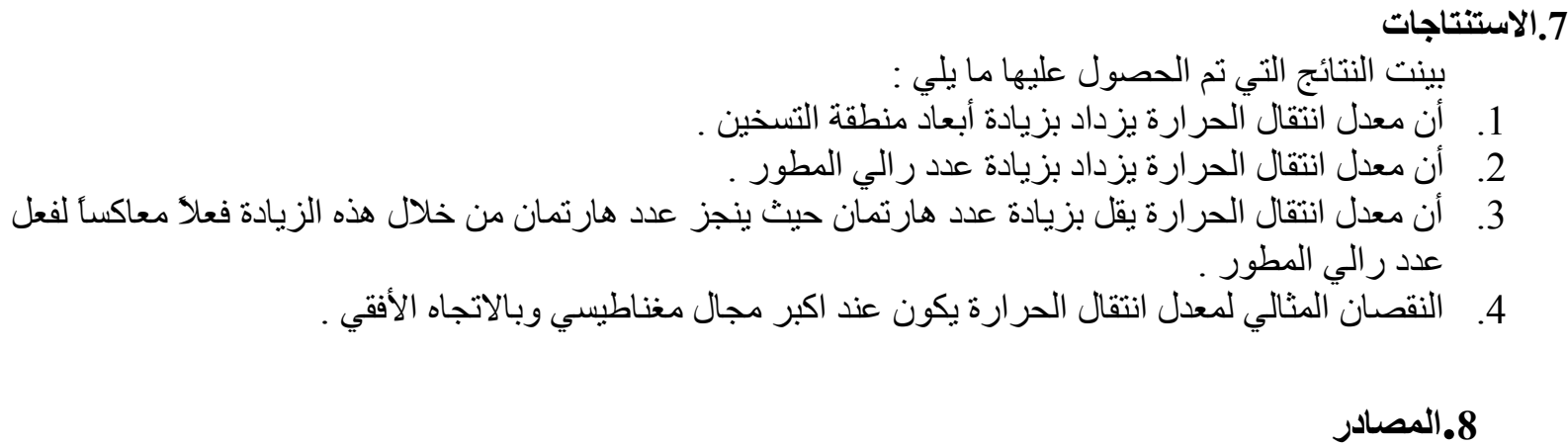

1- Kreith F.," Principles of Heat Transfer", Third Edition, Intext Educational Publishers, New york and London, Chapter 7, Page 383, 1973.

2- Kaluri R.S. Basak T. and Roy S.," Bejan's Heatlines and Numerical Visualization of Heat Flow and Thermal Mixing in Various Differentially Heated Porous Square Cavities ", Numerical Heat Transfer, Part A, 55: 487-516, 2009.

3- Grosan T. and Revnic C., Pop I. and Ingham D.B. "Magnetic field and internal heat generation effects on the free convection in a rectangular cavity filled with a porous medium", International Journal of Heat and Mass Transfer 52, 1525-1533, 2009.

4- Mansour M.A., Chamkha A.J., Mohamed R.A., Abd El-Aziz M.M. and Ahmed S.E. "MHD natural convection in an inclined cavity filled with a fluid saturated porous medium with heat source in the solid phase", Nonlinear Analysis: Modeling and Control, Vol. 15, No. 1, 55-70, 2010.

5- Saleh H., Roslan R. and Hashim I. " Natural convection in a porous trapezoidal enclosure with an inclined magnetic field", Computers and Fluids 47 ,155-164, 2011.

6- $\quad$ Ismaeel M.A." Partitioning and Magnetic Field Effects on Free Convection in a Square Cavity Filled with Porous Medium with Uniform Heat ", International Journal of Energy and Technology 4 (5), pp.1-11, 2012.

7- $\quad$ Kaluri R.S., Tanmay B. and Roy S." Bejan's Heatlines and Numerical Visualization of Heat Flow and Thermal Mixing in Various Differentially Heated Porous Square Cavities ", Numerical Heat Transfer, Part A, 55: 487-516, 2009.

8- Ismaeel M.A. "Numerical Study of Natural Convection Heat Transfer in an Inclined Square Porous Layer", Al-Rafidain EngineeringVol.16.NO. 3. Aug.2008.

9- Bejan, A. and Tien,C.L.," Natural Convection in a Horizontal Porous Medium Subjected to an End-to-End Temperature Difference", J. Heat Transfer, 100, pp.191198, 1978.

10- Chan B.K.C., Ivey C.M., and Barry J.M., "Natural Convection in Enclosed Porous Media with Rectangular Boundaries", J. Heat Transfer,2,pp.21-27,1970.

11- Burns P.J. ,Chow L.C., and Tien C.L. "Convection in Vertical Slot Filled with Porous Insulation", Int. J. Heat Mass Transfer,20,pp.919-926,1974.

12- Bejan A., "Natural Convection Heat Transfer in a Porous Layer with Internal Flow Obstruction ", Int. J. Heat Transfer ,26,pp.815-822,1983.

13- Dawood A. S."Steady Three -Dimensional Natural Convection in Porous Media Via Multi grid Method ", Ph.D. Dissertation, Dept. of Mech . Eng., Colorado State University, 1991.

$$
\text { تم اجراء البحث في كلية ألهنسة = جامعة ألموصل }
$$

